Research Paper

\title{
Stereotactic Body Radiotherapy (SBRT) Reirradiation for Recurrent Pancreas Cancer
}

\author{
Nergiz Dagoglu1, Mark Callery 2, James Moser ${ }^{2}$, Jennifer Tseng 2, Tara Kent ${ }^{2}$, Andrea Bullock ${ }^{3}$, Rebecca \\ Miksad $^{3}$, Joseph D. Mancias' ${ }^{1}$, Anand Mahadevan ${ }^{1, \bowtie}$ \\ 1. Department of Radiation Oncology; \\ 2. Department of Surgery; \\ 3. Department of Medical Oncology; Beth Israel Deaconess Medical Center and Harvard Medical School, Boston MA. \\ $\triangle$ Corresponding author: Anand Mahadevan MD, Department of Radiation Oncology, Beth Israel Deaconess Medical Center, 330 Brookline Ave, Boston MA \\ 02215. Tel: 6176672345, Fax: 6176674990
}

() Ivyspring International Publisher. Reproduction is permitted for personal, noncommercial use, provided that the article is in whole, unmodified, and properly cited. See http://ivyspring.com/terms for terms and conditions.

Received: 2015.07.20; Accepted: 2015.11.01; Published: 2016.01.10

\begin{abstract}
Objectives: After adjuvant or definitive radiation for pancreas cancer, there are limited conventional treatment options for recurrent pancreas cancer. We explored the role of (Stereotactic Body Radiotherapy) SBRT for reirradiation of recurrent pancreas Cancer.

Methods: This is a retrospective study of patients reirradiated with SBRT for recurrent pancreas cancer. All patients were deemed unresectable and treated with systemic therapy. Fiducial gold markers were used. CT simulation was performed with oral and IV contrast and patients were treated with respiratory motion tracking in the Cyberknife ${ }^{\mathrm{TM}}$ system.

Results: 30 patients (17 men and 13 women) with a median age of 67 years were included in the study. The median target volume was $41.29 \mathrm{cc}$. The median prescription dose was $25 \mathrm{~Gy}$ (24-36Gy) in a median of 5 fractions prescribed to a mean $78 \%$ isodose line. The median overall survival was 14 months. The 1 and 2 year local control was $78 \%$. The worst toxicity included 3/30(10\%) Grade III acute toxicity for pain, bleeding and vomiting. There was 2/30 (7\%) Grade III long-term bowel obstructions.

Conclusions: SBRT can be a useful and tolerable option for patients with recurrent pancreas cancer after prior radiation.
\end{abstract}

Key words: Pancreatic Cancer, SBRT (Stereotactic Body Radiotherapy), Reirradiation

\section{Introduction}

Pancreatic cancer is the fourth-leading cause of cancer-related mortality in the US (1). The natural history of pancreas cancer is characterized by poor outcomes at all stages and in patients with advanced disease $<5 \%$ of pancreatic cancer patients are still alive at 5 years from diagnosis (2). Despite advances in surgery, radiation therapy and systemic therapy most patients succumb to metastatic disease. Even after curative resection, prospective randomized adjuvant therapy trials have shown local failure rates ranging from $39-62 \%$, even in the presence of margin negative $\mathrm{R} 0$ resection (3-6).

While local failures may have an impact on sur- vival, they are also often symptomatic and debilitating, affecting quality of life. Only a small proportion of these patients are amenable for further surgical resection (7). In the setting of prior adjuvant radiation therapy, systemic therapy would be considered the standard; however response rates are poor and is associated with significant toxicity (8-10). Conventional external beam fractionated reirradiation is often feasible, but limited by the tolerance doses of normal tissue, making it risky (11).

An emerging treatment that has been successfully used in primary therapy and in the setting of reirradiation, due to its conformality and avoidance of 
normal structures, is Stereotactic Body Radiotherapy (SBRT). SBRT has been used as primary treatment for locally advanced pancreas cancer (12-19). As dose-limiting structures restrict reirradiation with conventional radiation, SBRT can be useful due its conformality in minimizing dose to critical normal structures around the tumor. SBRT reirradiation has been successfully used in Lung, Head and Neck, Spine and other cancers (20-23).

We report our experience in the use of SBRT for reirradiation for unresectable recurrent pancreatic cancer treated with prior radiation.

\section{Materials and Methods}

\section{Patients and Lesions}

Our institutional IRB approved database (DFHCC 09-451) was retrospectively reviewed. Patients treated with SBRT for recurrence after prior radiation for adenocarcinoma of the pancreas were included in this study. All patients were staged to exclude metastatic disease with a CT Torso. Clinical, radiological and biochemical (CA19-9) criteria were used to identify local recurrence and biopsy or PET scans were not mandated. Increase in size of local tumor in two dimensions or two consecutive elevations in CA19-9 with no demonstrable metastasis in torso CT staging and a positive PET scan when done were used to define local recurrence. All patients were systematically reviewed in a multidisciplinary pancreatic cancer conference and were deemed unresectable. These included patients after prior adjuvant radiation following pancreaticoduodenectomy and patients with progression after definitive conventional or SBRT for locally advanced pancreas cancer. All patients received salvage systemic therapy (gemcitabine, gemcitabine+nabpaclitaxel or FOLFIRNOX) at the discretion of the treating medical oncologist prior to and after initiation of the current SBRT study treatment.

\section{SBRT}

All patients who had prior adjuvant radiation or prior SBRT at our institution had fiducial gold markers placed at the time of surgery or under endoscopic ultrasound guidance prior to SBRT respectively. 3-5 gold fiducials were placed in all patients. Patients were simulated in supine position with Vacloc ${ }^{\mathrm{TM}}$ body immobilization system. Intravenous and barium contrast agent was used for the planning CT. The target volume was defined as gross tumor volume (GTV) and was contoured on axial CT images. The choice of fractionation was based on predetermined criteria including tumor volume, location of the tumor and prior radiation dose. Other factors, such as interval between prior RT, adjacent normal tissue, patients' performance and co morbidity were also taken into consideration. The irradiation dose was prescribed to the isodose line covering at least $95 \%$ of the target volume. Maximum point dose to the gastric and duodenal walls and the small bowel loops were kept at or below prescription dose. We have previously reported the treatment techniques used in this study $(14,15)$. Patients were treated on 3-5 consecutive days. Prophylactic prochlorperazine, H2 receptor antagonists and simethicone were used before and during the treatment.

\section{Follow up and statistical analysis}

Follow-up data included survival, date of progression and site of failure at the last follow up. Tumor response to treatment was evaluated by contrast-enhanced CT imaging. Local failure was defined as an increase in the sum of the longest diameter of target lesion. If a new lesion developed in the abdomen but outside the radiation field, it was interpreted as distant metastasis. Acute and late toxicities were defined as symptoms that develop within three months after SBRT or later, respectively. Local and distant progression rates and the lengths of overall survival were calculated from the date of treatment to the date of progression or death. The Kaplan-Meier method was used for calculating actuarial rates, using Graph Pad Prism version 5.00 software for Mac (Graph Pad Software, San Diego, CA; Windows; Microsoft, Seattle, WA).

\section{Results}

This study included 30 patients reradiated for recurrent pancreas cancer at our center between September 2005 and September 2013. 17 males and 13 females were treated. The median age at the time of reirradiation was 67 years (range 44 to 88 years). Median follow-up was 11 months (4 to 24 months).

Initial stages were as follows: Stage II or I in $14(48.1 \%)$ and Stage III in 16(51.9\%). All patients had previously received RT, either as part of their initial or salvage treatment after they recurred. 25 patients were irradiated as part of their initial treatment. Among those patients 9 of them underwent SBRT as their initial treatment. One patient had whole abdominal radiotherapy (WART) for ovarian cancer before she was diagnosed with pancreatic cancer. For the patients who were treated with SBRT the prior RT median initial dose was 24 Gy in 3 fractions (range, 24 to $30 \mathrm{~Gy}$ ) and for patients who were initially treated with External Beam Radiation Therapy (EBRT) was 50.4 Gy (range, 46.8-54 Gy). Time interval between initial RT and reirradiation with SBRT ranged from 4 to 316 months with a median of 18 months.

Sites of recurrence in previously resected pa- 
tients were the resection bed in 5, celiac or para aortic lymph node in 5, resection bed and lymph node in 2 and remnant pancreas in 1 . In locally advanced unresected patients the recurrences were progression in the head of the pancreas in 9, body in 3 and celiac lymph node in 1 . All patients had received initial chemotherapy. After recurrence, 14 patients had gemcitabine, 6 had FOLFOX and 2 had Erlotinib. 5 patients did not have additional chemotherapy.

Tumor volumes ranged from 12.03 to $315.72 \mathrm{cc}$ (median $41.29 \mathrm{cc}$ ). Median prescribed isodose was $78 \%$ (range $60-88 \%$ ). Total SBRT dose ranged from 24 to 36 Gy (median 25 Gy) in 3 to 5 fractions (median 5). Median conformality and heterogeneity indices were 1.23 and 1.28 respectively.

The patient and treatment characteristics are listed in Table 1. A representative treatment Plan is shown in Figure 1.

\section{Outcomes}

The overall median survival after reirradiation for recurrence was 14 months. The actuarial local progression free survival rate was $78 \%$. Four patients had only local progression, 6 had distant progression and 2 patients had both local and distant progression. Among the 8 patients with distant progression 3 had liver, 4 had peritoneal and 1 had lung metastasis. The actuarial 1-year and 2-year survival rates were 50\% and 5\% respectively. The 1-year and 2-year local control rates were $78 \%$. Kaplan Meir survival curves for Local Control and Overall Survival are shown in Figure 2 .

Table 1: Patient and Treatment Characteristics

\begin{tabular}{|c|c|c|}
\hline \multicolumn{2}{|c|}{ Patient and Treatment Characteristics. } & number \\
\hline \multicolumn{2}{|c|}{ Age (Range) } & $67(44-88)$ \\
\hline \multicolumn{2}{|l|}{$\operatorname{Sex}(m / f)$} & $17 / 13$ \\
\hline \multicolumn{2}{|c|}{ Time interval to reirradiation (months) } & $18(4-316)$ \\
\hline \multirow{7}{*}{$\begin{array}{l}\text { Initial Treat- } \\
\text { ment }\end{array}$} & Surgery + adjuvant Chemoradiation & $9(30 \%)$ \\
\hline & Induction chemotherapy +SBRT & $10(33.3 \%)$ \\
\hline & Definitive Chemoradiation & $4(13.3 \%)$ \\
\hline & Neoadjuvant Chemoradiation +surgery & $1(3.3 \%)$ \\
\hline & $\begin{array}{l}\text { Surgery + Adj Chemo + salvage } \\
\text { chemoradiation }\end{array}$ & $3(10 \%)$ \\
\hline & Radiotherapy & $1(3.3 \%)$ \\
\hline & Surgery+ salvage Chemoradiation & $2(6.7 \%)$ \\
\hline \multirow[t]{7}{*}{$\begin{array}{l}\text { SBRT Reirra- } \\
\text { diation }\end{array}$} & Treatment volume & $\begin{array}{l}41.29 \mathrm{cc} \\
(12.03-315.72)\end{array}$ \\
\hline & Prescribed isodose & $78 \%(60-88)$ \\
\hline & Total SBRT dose & $25(24-36)$ \\
\hline & No of fractions & $3(3-5)$ \\
\hline & Max dose & $32,47(29,27-40)$ \\
\hline & Conformality Index & $1,23(1,14-1,66)$ \\
\hline & Heterogeneity Index & $1,28(1,14-1,67)$ \\
\hline
\end{tabular}

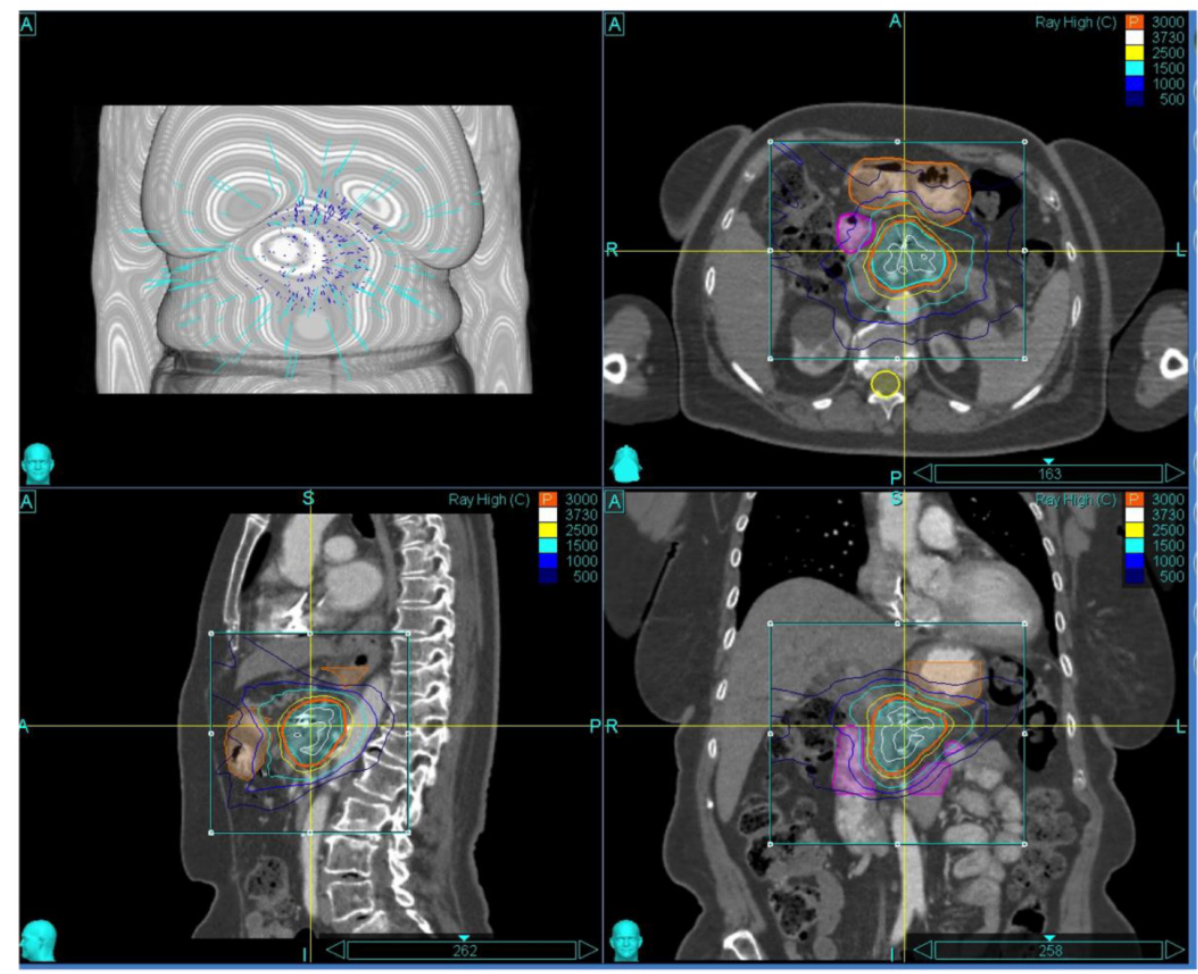

Figure 1: Representative Treatment Plan 

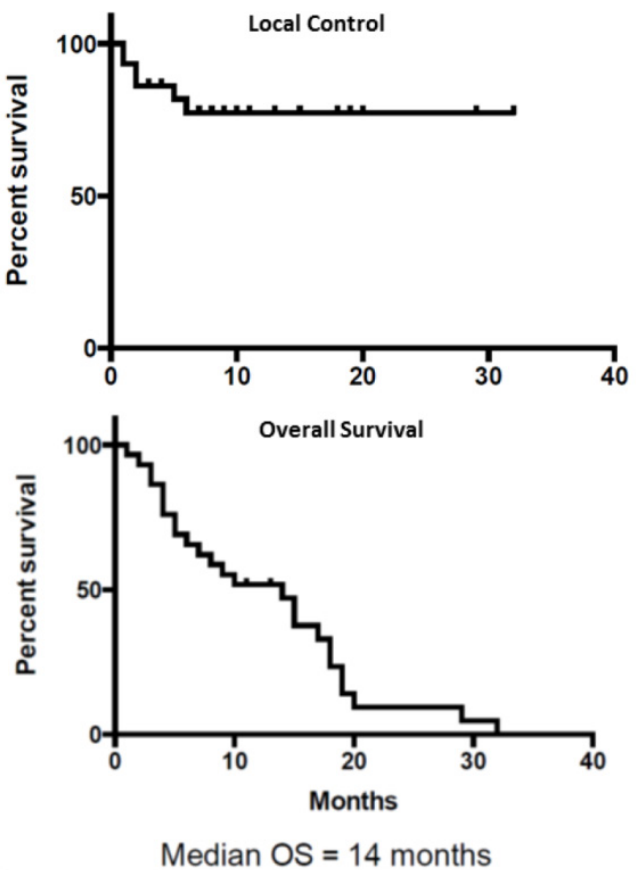

Figure 2: Local Control (Top Panel) and Overall Survival (Bottom Panel)

\section{Toxicity}

Treatment was well tolerated in the majority of patients. Sixteen patients $(60 \%)$ reported fatigue and four $(15 \%)$ reported nausea in the first four weeks of treatment. Two patients were hospitalized; one for gastroduodenal bleeding and vomiting and one patient was hospitalized for pain $(3 / 27 ; 11 \%$ Grade III acute Toxicity). There were 2 late Grade 3 GI complications (7\% Late Grade III Toxicity). Both patients developed bowel obstruction that resolved with conservative management not requiring surgery. Both patients were treated with SBRT as their previous treatment and both were under gemcitabine chemotherapy when the obstruction occurred. The time interval between the treatments was 23 and 42 months. Two other patient developed sub-acute ascites and both had negative cytology for malignant cells without any other evidence of progression.

\section{Discussion}

Management of recurrent pancreatic cancer is challenging (24). Therapeutic options are limited, particularly after prior radiation therapy. We report the utility of SBRT for reirradiation of locally recurrent pancreas cancer after prior radiation, with reasonable local control and modest survival.

While distant failure with or without local recurrence is a common after definitive local therapy for pancreas cancer including surgery $(25,26)$, there exists a subgroup of patients who develop isolated local recurrence without evidence of distant failure
$(4,27,28)$. Moreover, patients with isolated local recurrence after prior definitive local therapy appear to do better than patients with initially unresectable disease (29) or metastatic disease (30). This suggests patients may benefit from additional local therapy if feasible and safe. The NCCN (National Comprehensive Cancer Network) guidelines suggest that these patients be preferably treated as a part of clinical trials.

Surgery is not often feasible because of the extent of disease, prior radiation therapy and the poor performance status of these patients. In the largest series, Kleef et al for University of Heidelberg reported a series of 30 patients who underwent surgical exploration for recurrent pancreas cancer (31). Six patients had received prior radiation. Fifteen patients were able to undergo resection with 8 patients having R0/R1 resection. The remaining 15 patients had exploration and bypass. In addition, 8 patients who did not have prior adjuvant radiation received IORT (Intra Operative Radiation Therapy). The overall survival in these patients who were reoperated was 11.4 months. Patients who were able to undergo resection did better (medial overall survival 17 vs. 9.4 months). In addition patients with longer disease free interval from initial surgery appeared to do better. They also reported 1 postoperative death and postoperative morbidity in 5 patients. Zacharias et al from Strasbourg reported on 15 laparotomies for recurrent pancreas cancer (32). The perioperative mortality was $20 \%$; the post-operative morbidity was $40 \%$ and the median survival was 7.4 months. Our series of patients, who all received prior definitive radiation, compares well to selected patients undergoing repeat laparotomy and able to achieve resection.

After initial surgery and no post-operative radiation, Chemoradiation is a viable option for isolated local recurrences. Wilkowski et al utilized 45 Gy 3D-conformal radiation therapy with concurrent chemotherapy (continuous infusion 5FU, gemcitabine or cisplatinum) in 18 patients with isolated local recurrences (33). They reported $80 \%$ response rate with a $35 \%$ complete response, $37.5 \%$ partial response and $25 \%$ with stable disease. The median progression free survival was 14.7 months and the median overall survival was 17.5 months. There was no grade 3 or 4 gastrointestinal toxicity. Similarly, Nakamura and colleagues used 54 Gy EBRT with concurrent gemcitabine or S1 (11). The 1-year overall survival, local control, and progression-free survival rates were $69 \%$, $67 \%$, and $32 \%$, respectively. The median overall survival and progression-free survival rates were 15.9 and 6.9 months.

In the setting of prior adjuvant or definitive RT, systemic therapy is often considered the standard of 
care for local recurrence when reoperation is not feasible or safe $(9,8,10,34)$. Reirradiation with conventional EBRT carries significant risk for morbidity and mortality due to dose limiting toxicity from prior radiation. In this series we have explored the role SBRT as additional local therapy in this setting.

Stereotactic Body Radiotherapy (SBRT) has been successfully used in the primary therapy and in the setting of reirradiation for a variety of cancer sites due to its conformality and avoidance of normal structures $(13-15,20)$. Additionally, short courses of SBRT allow for standard of care chemotherapy regimens to be administered without interruption.

SBRT has been shown to be feasible after conventional EBRT as a planned boost to intensify local therapy for pancreatic cancer, particularly in the locally advanced unresectable setting and for positive margins. A study from Stanford included 19 patients who were treated with a protocol of 45 Gy intensity-modulated radiation therapy (IMRT) with concurrent 5-FU chemotherapy followed by a 25 Gy SBRT boost in one fraction to the primary tumor (35). Sixteen patients completed the planned therapy. Four patients experienced grade 2 toxicity and 2 patients experienced grade 3 toxicity. Fifteen of these 16 patients were free from local progression until death. Median overall survival was 33 weeks. They reported a high local control rate of $94 \%$ but with the accompanying toxicity and high systemic failure rates. Similarly Seo et al reported a retrospective series of 30 patients treated with a similar strategy involving conventional EBRT for total dose of 40 Gy delivered in 20 fractions followed by a single fraction of 14, 15, 16, or 17 Gy SBRT administered as a boost without a break (36). Twenty-one patients received chemotherapy. Five patients were lost to follow-up. Local progression only was identified in 3 of the $25(12 \%)$, and both local and distant metastasis in 8 of the $25(32 \%)$. Of the 30 patients, $20(66.7 \%)$ exhibited Grade 1 or 2 acute toxicity (nausea, vomiting, and/or pain) for a short period. One patient required bypass surgery because of the development of a duodenal obstruction 3 months after SBRT treatment.

Two other series have reported the utility of SBRT after prior definitive EBRT. The University of Pittsburgh has reported a series of 71 patients who had undergone SBRT for locally advanced pancreas cancer. Fifteen of these patients were receiving SBRT in the reirradiation setting after a median prior EBRT dose of 45 Gy. The median SBRT dose was 24 Gy (18-25 Gy), given in a single fraction for 67 patients and fractionated in 4 patients. The outcomes of patients who were reirradiated were not reported separately (19). Lominska et al from Georgetown University reported a series of 28 patients who received
SBRT after a median prior EBRT dose of 50.4 Gy (41.40-70.20Gy) (37). 11 of these patients were treated with a hypofractionated boost with SBRT delivered within 2 months of completing EBRT. The remaining 17 patients underwent salvage SBRT after imaging demonstrated local recurrence or progression. For all patients, median total SBRT dose was 22.5 Gy (20-30) with a median dose per fraction being 7 Gy (4-8) and median number of fractions being $3(3-5)$. They did not report outcomes for the two subgroups and the outcomes for elective versus salvage SBRT for recurrences after prior EBRT is unclear. Overall, 12 of 14 $(85.7 \%)$ evaluable patients were free from local progression. The median overall survival was 5.9 months, with $18 \%$ survival and $70 \%$ freedom from local progression at one year. Toxicity consisted of one case of acute grade 2 nausea/vomiting, and 2 cases of grade 3 late GI toxicity, one being bowel obstruction and the other perforation both of which resolved with conservative management. Both patients were treated with 21 Gy in three fractions, and had received 50.4 Gy to the abdomen previously.

Our series exclusively reports on outcomes in the difficult clinical setting of local recurrence after prior definitive radiation for pancreas cancer. The median survival of 14 months and the local control of $78 \%$ compares favorably with the previously reported results in this scenario. Treatment related toxicity is of significant concern with reirradiation. The $11 \%$ acute grade 3 toxicity, with resolution and the eventual $7 \%$ long term toxicity appears reasonable in this difficult situation.

\section{Conclusion}

SBRT reirradiation for recurrent pancreas cancer, due to its conformality and avoidance of dose limiting critical structures, seems feasible, effective and tolerable. Future prospective studies are needed to define the role of SBRT reirradiation for local recurrences in the setting of systemic therapy.

\section{Competing Interests}

The authors have declared that no competing interest exists.

\section{References}

1. Siegel R, Ma J, Zou Z, Jemal A. Cancer statistics, 2014: Cancer Statistics, 2014. CA Cancer J Clin. 2014 Jan;64(1):9-29.

2. Simianu VV, Zyromski NJ, Nakeeb A, Lillemoe KD. Pancreatic cancer: Progress made. Acta Oncol. 2010 May;49(4):407-17.

3. Gastrointestinal Tumor Study Group. Further evidence of effective adjuvant combined radiation and chemotherapy following curative resection of pancreatic cancer. Cancer. 1987;59(12):2006-10.

4. Neoptolemos JP, Stocken DD, Friess H, Bassi C, Dunn JA, Hickey H, et al. A randomized trial of chemoradiotherapy and chemotherapy after resection of pancreatic cancer. N Engl J Med. 2004 Mar 18;350(12):1200-10.

5. Regine WF, Winter KA, Abrams RA, Safran H, Hoffman JP, Konski A, et al. Fluorouracil vs gemcitabine chemotherapy before and after fluorouracil-based chemoradiation following resection of pancreatic adenocarcinoma: a randomized controlled trial. JAMA. 2008 Mar 5;299(9):1019-26. 
6. Oettle H, Post S, Neuhaus P, Gellert K, Langrehr J, Ridwelski K, et al. Adjuvant chemotherapy with gemcitabine vs observation in patients undergoing curative-intent resection of pancreatic cancer: a randomized controlled trial. JAMA. 2007 Jan 17;297(3):267-77.

7. Miyazaki M, Yoshitomi H, Shimizu H, Ohtsuka M, Yoshidome H, Furukawa $\mathrm{K}$, et al. Repeat pancreatectomy for pancreatic ductal cancer recurrence in the remnant pancreas after initial pancreatectomy: Is it worthwhile? Surgery. 2014 Jan;155(1):58-66.

8. Conroy T, Desseigne F, Ychou M, Bouché O, Guimbaud R, Bécouarn Y, et al. FOLFIRINOX versus gemcitabine for metastatic pancreatic cancer. N Engl J Med. 2011;364(19):1817-25.

9. Burris HA, Moore MJ, Andersen J, Green MR, Rothenberg ML, Modiano MR, et al. Improvements in survival and clinical benefit with gemcitabine as first-line therapy for patients with advanced pancreas cancer: a randomized trial. J Clin Oncol. 1997 Jun;15(6):2403-13.

10. Lee MG, Lee SH, Lee SJ, Lee YS, Hwang J-H, Ryu JK, et al. 5-Fluorouracil/Leucovorin Combined with Irinotecan and Oxaliplatin (FOLFIRINOX) as Second-Line Chemotherapy in Patients with Advanced Pancreatic Cancer Who Have Progressed on Gemcitabine-Based Therapy. Chemotherapy. 2013;59(4):273-9.

11. Nakamura A, Itasaka S, Takaori K, Kawaguchi Y, Shibuya K, Yoshimura M, et al. Radiotherapy for patients with isolated local recurrence of primary resected pancreatic cancer: Prolonged disease-free interval associated with favorable prognosis. Strahlenther Onkol. 2014 May;190(5):485-90.

12. Koong AC, Le QT, Ho A, Fong B, Fisher G, Cho C, et al. Phase I study of stereotactic radiosurgery in patients with locally advanced pancreatic cancer. Int J Radiat Oncol Biol Phys. 2004 Mar 15;58(4):1017-21.

13. Chang DT, Schellenberg D, Shen J, Kim J, Goodman KA, Fisher GA, et al. Stereotactic radiotherapy for unresectable adenocarcinoma of the pancreas. Cancer. 2009 Feb 1;115(3):665-72.

14. Mahadevan A, Miksad R, Goldstein M, Sullivan R, Bullock A, Buchbinder E et al. Induction gemcitabine and stereotactic body radiotherapy for locally advanced nonmetastatic pancreas cancer. Int J Radiat Oncol Biol Phys. 2011;

15. Mahadevan A, Jain S, Goldstein M, Miksad R, Pleskow D, Sawhney M, et al. Stereotactic body radiotherapy and gemcitabine for locally advanced pancreatic cancer. Int J Radiat Oncol Biol Phys. 2010;78(3):735-42.

16. Didolkar MS, Coleman CW, Brenner MJ, Chu KU, Olexa N, Stanwyck E, et al. Image-guided stereotactic radiosurgery for locally advanced pancreatic adenocarcinoma results of first 85 patients. J Gastrointest Surg Off J Soc Surg Aliment Tract. 2010 Oct;14(10):1547-59.

17. Goyal K, Einstein D, Ibarra RA, Yao M, Kunos C, Ellis R, et al. Stereotactic Body Radiation Therapy for Nonresectable Tumors of the Pancreas. J Surg Res [Internet]. 2011 Aug [cited 2011 Nov 27]; Available from: http://linkinghub.elsevier.com/retrieve/pii/S0022480411006743

18. Hoyer M, Roed $\mathrm{H}$, Sengelov L, Traberg A, Ohlhuis L, Pedersen J, et al. Phase-II study on stereotactic radiotherapy of locally advanced pancreatic carcinoma. Radiother Oncol J Eur Soc Ther Radiol Oncol. 2005 Jul;76(1):48-53.

19. Rwigema J-CM, Parikh SD, Heron DE, Howell M, Zeh H, Moser AJ, et al. Stereotactic Body Radiotherapy in the Treatment of Advanced Adenocarcinoma of the Pancreas. Am J Clin Oncol [Internet]. 2010 Mar 19; Available from: http://www.ncbi.nlm.nih.gov/pubmed/20308870

20. Mahadevan A, Floyd S, Wong E, Jeyapalan S, Groff M, Kasper E. Stereotactic body radiotherapy reirradiation for recurrent epidural spinal metastases. Int $\mathrm{J}$ Radiat Oncol Biol Phys. 2011 Dec 1;81(5):1500-5.

21. Valakh V, Miyamoto C, Micaily B, Chan P, Neicu T, Li S. Repeat stereotactic body radiation therapy for patients with pulmonary malignancies who had previously received SBRT to the same or an adjacent tumor site. J Cancer Res Ther. 2013 Dec;9(4):680-5.

22. Lartigau EF, Tresch E, Thariat J, Graff P, Coche-Dequeant B, Benezery K, et al. Multi institutional phase II study of concomitant stereotactic reirradiation and cetuximab for recurrent head and neck cancer. Radiother Oncol J Eur Soc Ther Radiol Oncol. 2013 Nov;109(2):281-5.

23. Patel PR, Salama JK. Reirradiation for recurrent head and neck cancer. Expert Rev Anticancer Ther. 2012 Sep;12(9):1177-89.

24. Kyriazanos ID, Tsoukalos GG, Papageoroiou G, Verigos KE, Miliadis L, Stoidis CN. Local recurrence of pancreatic cancer after primary surgical intervention: How to deal with this devastating scenario? Surg Oncol. 2011 Dec;20(4):e133-e142.

25. Sperti C, Pasquali C, Piccoli A, Pedrazzoli S. Recurrence after resection for ductal adenocarcinoma of the pancreas. World J Surg. 1997;21(2):195-200.

26. Hernandez JM, Morton CA, Al-Saadi S, Villadolid D, Cooper J, Bowers C, et al. The natural history of resected pancreatic cancer without adjuvant chemotherapy. Am Surg. 2010 May;76(5):480-5.

27. Van Laethem J-L, Hammel P, Mornex F, Azria D, Van Tienhoven G, Vergauwe $P$, et al. Adjuvant Gemcitabine Alone Versus Gemcitabine-Based Chemoradiotherapy After Curative Resection for Pancreatic Cancer: A Randomized EORTC-40013-22012/FFCD-9203/GERCOR Phase II Study. J Clin Oncol. 2010 Oct 10;28(29):4450-6.

28. Van den broeck A, Sergeant G, Ectors N, Van Steenbergen W, Aerts R, Topal B. Patterns of recurrence after curative resection of pancreatic ductal adenocarcinoma. Eur J Surg Oncol EJSO. 2009 Jun;35(6):600-4

29. Xue P, Kanai M, Mori Y, Nishimura T, Uza N, Kodama Y, et al. Comparative Outcomes Between Initially Unresectable and Recurrent Cases of Advanced Pancreatic Cancer Following Palliative Chemotherapy. Pancreas. 2014;43(3):411-6.
30. Hashimoto $\mathrm{K}$, Ueno $\mathrm{H}$, Ikeda $\mathrm{M}$, Kojima $\mathrm{Y}$, Hagihara A, Kondo S, et al. Do Recurrent and Metastatic Pancreatic Cancer Patients Have the Same Outcomes with Gemcitabine Treatment? Oncology. 2009;77(3-4):217-23.

31. Kleeff J, Reiser C, Hinz U, Bachmann J, Debus J, Jaeger D, et al. Surgery for Recurrent Pancreatic Ductal Adenocarcinoma. Ann Surg. 2007 Apr;245(4):566-72.

32. Zacharias T, Oussoultzoglou E, Jaeck D, Pessaux P, Bachellier P. Surgery for Recurrence of Periampullary Malignancies. J Gastrointest Surg. 2009 Apr;13(4):760-7.

33. Wilkowski R, Thoma M, Bruns C, Duhmke E, Heinemann V. Combined chemoradiotherapy for isolated local recurrence after primary resection of pancreatic cancer. Jop. 2006;7(1):34-40.

34. Gresham GK, Wells GA, Gill S, Cameron C, Jonker DJ. Chemotherapy regimens for advanced pancreatic cancer: a systematic review and network meta-analysis. BMC Cancer. 2014 Jun 27;14(1):471.

35. Koong AC, Christofferson E, Le Q-T, Goodman KA, Ho A, Kuo T, et al. Phase II study to assess the efficacy of conventionally fractionated radiotherapy followed by a stereotactic radiosurgery boost in patients with locally advanced pancreatic cancer. Int J Radiat Oncol Biol Phys. 2005 Oct 1;63(2):320-3.

36. Seo Y, Kim M-S, Yoo S, Cho C, Yang K, Yoo H, et al. Stereotactic Body Radiation Therapy Boost in Locally Advanced Pancreatic Cancer. Int J Radiat Oncol. 2009 Dec;75(5):1456-61.

37. Lominska CE, Unger K, Nasr NM, Haddad N, Gagnon G. Stereotactic body radiation therapy for reirradiation of localized adenocarcinoma of the pancreas. Radiat Oncol. 2012;7(1):74. 